\title{
Grant DE-FG02-86ER45239
}

DE91 000267

\section{Progress summary}

(This report prepared in September, 1990)

In this second period of support we have concentrated exclusively on three subjects, namely, (1) rare earths, bulk and surfaces; (2). ultrathin films of strained metallic phases; and (3) improvement of experimental facilities.

In the area of rare earths we have analyzed the photoemission data collected at beamline U7B of the National Synchrotron Light Source from a single-crystal platelet of $\mathrm{Tb}(0001)$. We have been able to map, for the first time in any rare-earth metal, the full dispersed $6 s-5 d_{3 z^{2}-1}$-type $\Delta_{1}$ band along the central six-fold axis of the Brillouin zone (the $\Gamma \Delta A$ line). We determined the two critical points of the $\Delta_{1}$ band at $\Gamma_{1}^{+}=-6.9 \mathrm{eV}$ and $\Gamma_{4}^{-}=-3.6 \mathrm{eV}$. Collaboration with N.E. Christensen of the Institute of Physics in Aarahus in Denmark enabled us to compare the experimental band structure with the result of a relativistic linear-muffin-tin-orbital calculation. Dispersion and width of the initial $\Delta_{1}$ band are well reproduced by theory, but the absolute energy position of the experimental band deviates by $-1.5 \mathrm{eV}$ from theory.

LEED experiments on the same $\mathrm{Tb}(0001)$ surface produced, after considerable time-consuming cleaning procedures, excellent LEED patterns with sharp spots and low background. But Auger spectroscopy still revealed the presence of substantial amounts of $\mathrm{Fe}$ in the surface region. The Fe impurity was incorporated in the crystal during the growth process (the crystals were purchased from the Ames Laboratory in Iowa, the sole source of rare-earth single crystals in the USA) and always segregated on the surface during the annealing process that must follow the ion-bombardment step of the cleaning sequence. Very probably because of the Fe impurity, we were

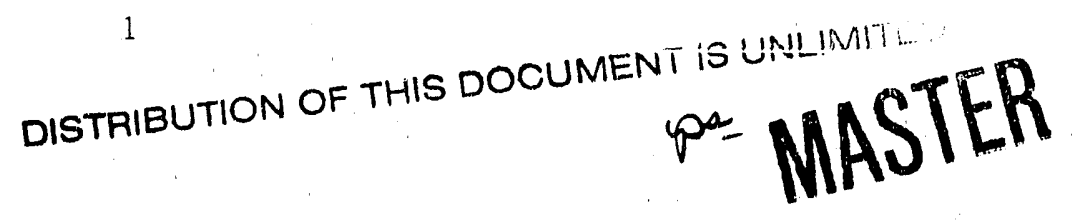


unable to fit the experimental LEED I(V) curves with any theoretical structural model compatible with the basal-plane geometry.

One possible way out of this dilemma was to try and grow thin films of $\mathrm{Tb}$ on a suitable substrate, which turned out to be $\mathrm{W}\{110\}$. We were successful in this attempt insofar as films could be grown that contained no Fe impurity, but the LEED patterns obtained from these thin films were notably poorer in sharpness and background than those obtained from the bulk single crystal. Nevertheless, LEED I(V) curves could be collected, and they turned out to be quite different from those collected earlier from the bulk single crystal. Intensity calculations, now in progress, are promisingly closer to experiment than before, but the fit is still not quite satisfactory. This work is still in progress.

The study of epitaxial strained phases was devoted to confirmation of the remarkable growth of highly strained $\mathrm{Cu}\{001\}$ on $\mathrm{Pd}\{001\}$. We did so by examining the growth of $\mathrm{Cu}\{001\}$ films on a $\mathrm{Pt}\{001\}$ surface-Pt $\{001\}$ has a surface lattice constant $(2.78 \AA)$ which is a bit larger than that of $\operatorname{Pd}\{001\}(2.75 \AA)$ and hence provides a somewhat larger strain to fcc $\mathrm{Cu}\{001\}$ (2.55 ) than the Pd counterpart. We found that $\mathrm{Cu}$ films could be grown to thicknesses of about 10 atomic layers with a strain just about equal to that found on films grown on $\operatorname{Pd}\{001\}$. The phenomenon is therefore confirmed. This work is being been written up and will be submitted for publication in the near future.

In the same light we examined the growth of $b c c \mathrm{Fe}$ on $\mathrm{Ag}\{001\}$ and on $\mathrm{Pd}\{\{001\}$. In the former case we confirmed good epitaxy, but we uncovered two new aspects of this problem. One is that while deposition of $\mathrm{Fe}$ on clean $\mathrm{Ag}\{001\}$ results at first in a strong deterioration of the LEED pattern (an observation that was made 
and reported by a few other workers earlier), prolongued deposition restores a fairly good LEED pattern, demonstrating pseudomorphic epitaxy of rather thick films. In fact, we analyzed such films to confirm quantitatively that they were indeed $\alpha-\mathrm{Fe}$ with only very slightly distorted $b c c$ structure. The second new result is that these observations are not consistent with the conclusions reached by other workers on the same system on the basis of RHEED observations. This discrepancy between LEED and RHEED observations is intriguing and may have far-reaching consequences about the widely accepted interpretation of RHEED intensity oscillations as signalling layer-by-layer growth. The results of this work have been have been condensed in the form of a Rapid Communication which has been submitted to the Physical Review for publication.

The Fe/Pd $\{001\}$ system is peculiar in a different way. In this case Fe is strained (about $4.5 \%$ ), but can nevertheless be grown to thicknesses of 60 and more atomic layers. The peculiarity lies in the fact that the interlayer spacings in the surface region are found to depend upon film thickness-a rather new effect which is not well understood at the present time.

In the area of improvement of experimental facilities we have devoted some time to the redesign of our (already successfully tested) 3-axes sample manipulator with the idea of using piezo-robots instead of mechanical linkages. The redesign aims at extending the range of specimen tilt (which is limited to about $55^{\circ}$ in the manipulator now in use) and improving the operation and the reliability of the azimuthal rotation. The design is almost completed at the time of writing and we would hope to be able to use the new manipulator early in 1991 . 
II. Research proposed for the next period of support

We plan to continue devoting most of our efforts to the studies of rare-earth surfaces by both LEED and photoemission. We are particularly concerned with the role played by $\mathrm{Fe}$ in the surface region of single crystals of the rare earths, not only for scientific but also for technological reasons, as Fe seems to be a rather common impurity in the rare earths and alloys of $\mathrm{Fe}$ with rare-earth metals have useful and interesting magnetic properties.

We also plan to continue our studies of the properties of strained and metastable phases of metals in the form of epitaxial thin films. Thus, we will re-examine the question of bcc copper by experimenting with the growth of $\mathrm{Cu}$ on $\mathrm{Ag}\{001\}$, and we will extend the investigation of strained $f c c \mathrm{Cu}$ by looking at its growth on $\operatorname{Pd}\{111\}$. 

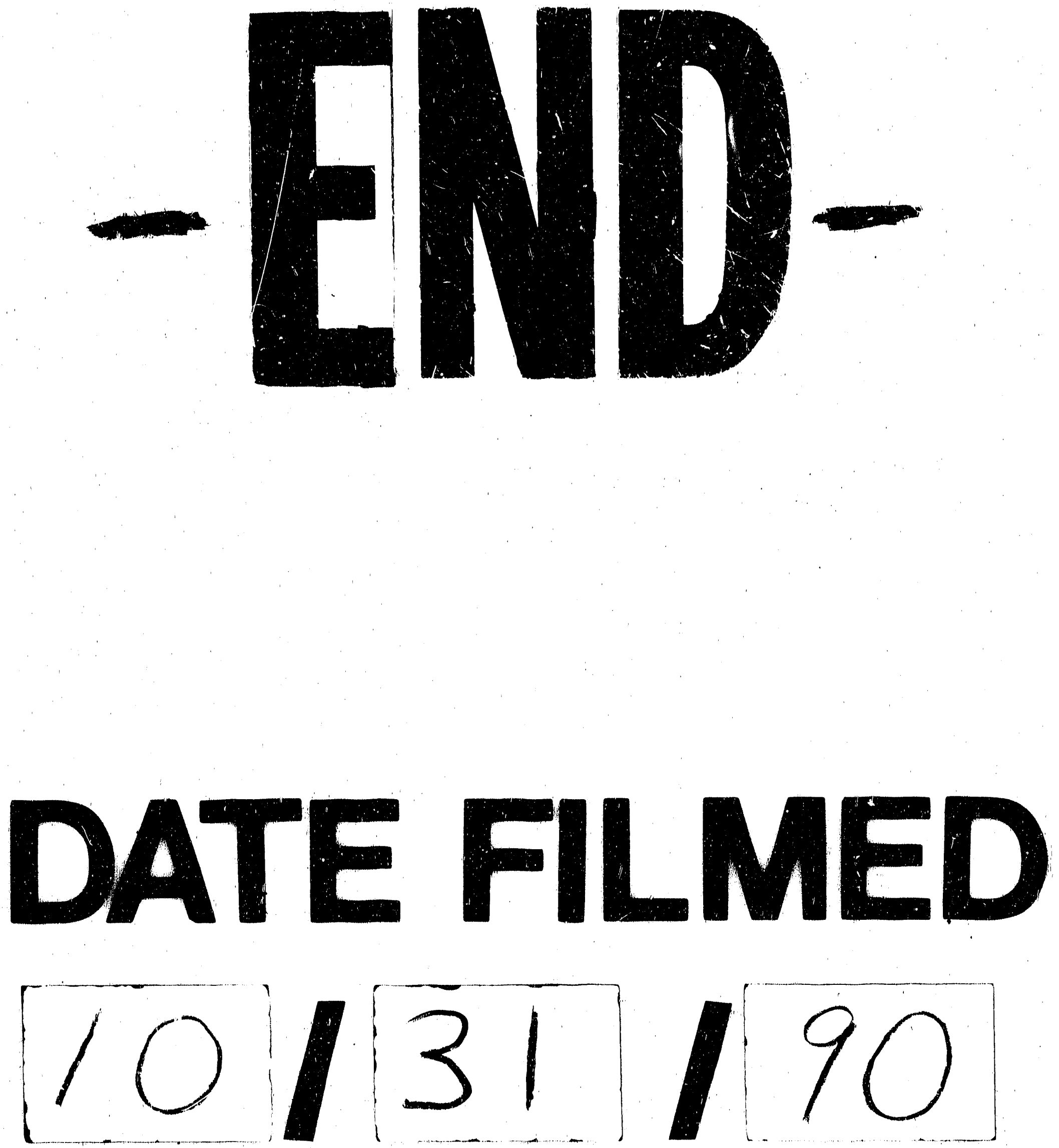
